\title{
Solar flares: self-organization of active region to the critical state
}

\author{
A.R.Osokin ${ }^{1}$, A.V.Podlazov ${ }^{2}$, V.A.Chernetsky ${ }^{3}$ and M.A.Livshits ${ }^{3}$ \\ ${ }^{1}$ Sternberg State Astronomical Institute, Moscow State University, 119899 Moscow, Russia \\ ${ }^{2}$ M.V.Keldysh Institute for Applied Mathematics Russian Academy of Sciences Miusskaya sq., \\ 4. 125047 Moscow, Russia \\ ${ }^{3}$ Institute of Terrestrial Magnetism, Ionosphere and Radio Wave Propagation Russian \\ Academy of Sciences 142190 Troitsk, Russia
}

\begin{abstract}
Results of modern multi-wavelength observations of large solar non-stationary events are briefly summarized. Such events consist of a puls, an eruption (surge or CME) and a longliving system of giant arches.

Observations demonstrate often that the pulse arises as a result of "avalanche" of interaction of small-scale magnetic elements, while the dynamic system transforms into a state of selforganizing criticality (SOC) on the post-eruptive phase. We developed new code for the avalanche model where it is possible to consider a transition of the active region as a dynamic system in a state of SOC. Derived solutions show, firstly, that the avalanche is naturally developed nearby spots during the pulse. Secondly, during long-duration events, occuring along the neutral line of the longitudinal magnetic field, behaviour of the large-scale coronal magnetic field is characterized by a state of SOC.
\end{abstract}

Flares occur in regions which are characterized by a definite level of dynamical activity. There is adopted point of view that flares appear when new magnetic flux emerges. Weak small-scale dynamic events associated with this appearance have progress either in superpenumbra of some sunspots or along neutral line of longitudinal magnetic field. There are reasons to think these two types are associated with appearance of impulses and two-ribbons flares correspondently.

According to modern ideas quite large flare consists of one or a few impulsive events, plasma ejection and further development of giant post eruptive loops. Observations of flare activity resulted in establishing the power-law in the distribution the flare number (or the flare frequency) from their energy. This is the reason to use the theory of self organized criticality. For the first time it was made by Lu and Hamilton 1.

In the active region, the energy of the magnetic fields is large enough. It exceeds significantly the energy losses during large solar flares. We tried to determine conditions when dissipation of the part of the energy occurs in a few hours. Probably relationships between excitation and relaxation of parameters established in this time interval in a dynamic system, which represents the active region; i.e. a large flare is either a probable process, arising in a self-organized systems in a critical state. This concept differs from our idea by solution of soliton hypothesis.

Our model 2 is a simple cellular automaton representing the active region by means of a two-dimensional rectangular lattice with periodic boundary conditions. Each lattice cell can contain one or more magnetic elements (i.e. small - scale magnetic tubes) or it can be empty.

Energy dissipation occurs if in any cell there are elements of opposite marks (that corresponds to contact of small magnetic tubes of opposite directed field). The result 


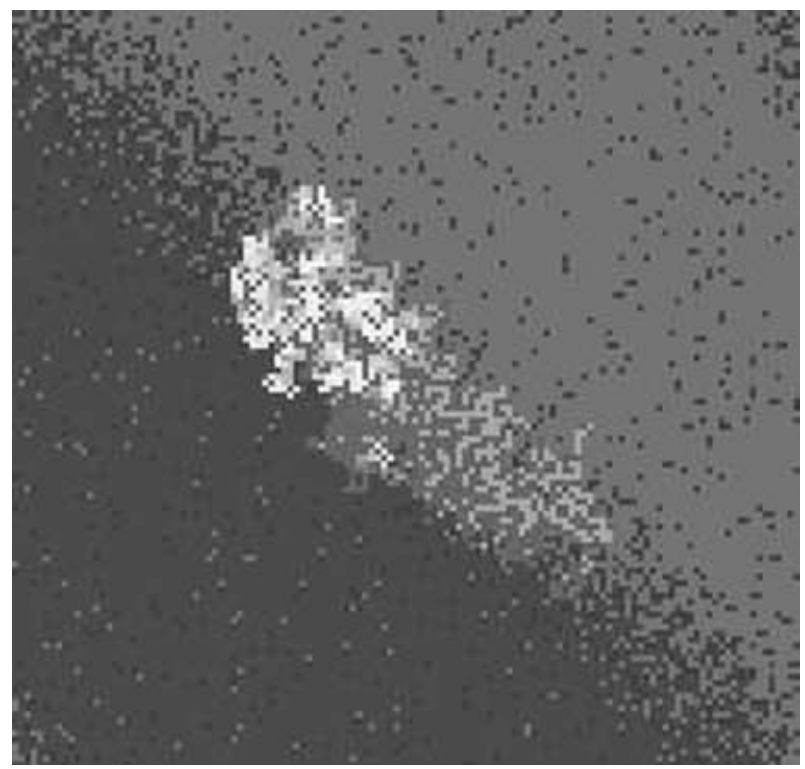

Figure 1. Development of avalanche/flare among the "neutral line" between the ribbon hills of large-scale magnetic fields. White pixels are on sites where avalanche is developing, while grey ones - where it has finished.

is moving "elements" to the next cells. There can be an avalanche of the individual processes under some conditions. It is important the physical transparency of the model is possible for additional features of magnetic fields.

Such avalanche is nothing else than flare.

Importing thing was the visualization of modelling results of 1 and 2 programs.

As a result of this simulation we obtained the Power low distribution of the flare frequency from their energy and we used it for testing of new modelling.

In the start of the calculation, when distribution of elements was uniform, avalanches occurred in different places. This two-ribbon character of the flare, is realized rather rarely in our modelling.

In the second code we took into account that flares occur between hills of the large-scale magnetic field. It is modelled by injection of one-sign elements into one region of the site simulated, while opposite-sign are injected into another place, i.e. in a result there are two zones and the neutral line between them. It was more convenient to make simulations for regions of alternate magnetic polarity - tore-like surface to exclude boundary effects. Otherwise, the magnetic flux of a ribbon becomes close to a flux of the large sunspot, and the distance between ribbon is close to that between large sunspots of opposite polarity.

Therefore, if we add large-scale magnetic field to our first model based on self-organized criticality theory, large flares start from small avalanche and propagate along the neutral line. Probability character of flare as a whole development conserves; thereby the energy of first impulse effects on the further process.

The work is supported by RFBR under grants numbers 02-02-17086 and 02-02-17928.

\section{References}

Lu, E. T., Hamilton, R. J,: 1991, ApJ 380, L89 - L92

Podlazov, A. V., Osokin, A. R.: 2002, A\&SpSci. 282, $221-226$ 